\title{
TRACE INEQUALITIES FOR MATRICES
}

\author{
KHALID SHEBRAWI ${ }^{\circledR}$ and HUSSIEN ALBADAWI
}

(Received 23 February 2012; accepted 20 June 2012)

\begin{abstract}
Trace inequalities for sums and products of matrices are presented. Relations between the given inequalities and earlier results are discussed. Among other inequalities, it is shown that if $A$ and $B$ are positive semidefinite matrices then
\end{abstract}

$$
\operatorname{tr}(A B)^{k} \leq \min \left\{\|A\|^{k} \operatorname{tr} B^{k},\|B\|^{k} \operatorname{tr} A^{k}\right\}
$$

for any positive integer $k$.

2010 Mathematics subject classification: primary 15A18; secondary 15A42, 15A45.

Keywords and phrases: trace inequalities, eigenvalues, singular values.

\section{Introduction}

Let $M_{n}(\mathbb{C})$ be the algebra of all $n \times n$ matrices over the complex number field. The singular values of $A \in M_{n}(\mathbb{C})$, denoted by $s_{1}(A), s_{2}(A), \ldots, s_{n}(A)$, are the eigenvalues of the matrix $|A|=\left(A^{*} A\right)^{1 / 2}$ arranged in such a way that $s_{1}(A) \geq s_{2}(A) \geq \cdots \geq s_{n}(A)$. Note that $s_{i}^{2}(A)=\lambda_{i}\left(A^{*} A\right)=\lambda_{i}\left(A A^{*}\right)$, so for a positive semidefinite matrix $A$, we have $s_{i}(A)=\lambda_{i}(A)(i=1,2, \ldots, n)$.

The trace functional of $A \in M_{n}(\mathbb{C})$, denoted by $\operatorname{tr} A$ or $\operatorname{tr}(A)$, is defined to be the sum of the entries on the main diagonal of $A$ and it is well known that the trace of a matrix $A$ is equal to the sum of its eigenvalues, that is, $\operatorname{tr} A=\sum_{j=1}^{n} \lambda_{j}(A)$. Two principal properties of the trace are that it is a linear functional and, for $A, B \in M_{n}(\mathbb{C})$, we have $\operatorname{tr}(A B)=\operatorname{tr}(B A)$. Trace inequalities are used in many applications such as control theory, quantum information theory, computational statistics and communication systems; see, for example, [5]. For the theory of trace functionals and their applications the reader is referred to [10].

It is well known that the trace functional is submultiplicative, that is, for positive semidefinite matrices $A$ and $B$ in $M_{n}(\mathbb{C})$,

$$
0 \leq \operatorname{tr}(A B) \leq \operatorname{tr} A \operatorname{tr} B
$$

(C) 2012 Australian Mathematical Publishing Association Inc. 0004-9727/2012 \$16.00 
Yang et al. [13] show further that for any integer $m$,

$$
\operatorname{tr}(A B)^{m} \leq\left(\operatorname{tr} A^{2 m}\right)^{1 / 2}\left(\operatorname{tr} B^{2 m}\right)^{1 / 2} .
$$

Clearly, (1.2) implies (1.1) since $\left(\operatorname{tr} A^{2}\right)^{1 / 2} \leq \operatorname{tr} A$ for any matrix $A$. Also, Ando [1] proved a very strong form of Young's inequality - it was shown that if $A$ and $B$ are in $M_{n}(\mathbb{C})$, then there is a unitary matrix $U$ such that

$$
\left|A B^{*}\right| \leq U\left(\frac{1}{p}|A|^{p}+\frac{1}{q}|B|^{q}\right) U^{*},
$$

where $p, q$ are positive real numbers satisfying $1 / p+1 / q=1$, which immediately gives

$$
\operatorname{tr}\left|A B^{*}\right| \leq \frac{\operatorname{tr}|A|^{p}}{p}+\frac{\operatorname{tr}|B|^{q}}{q} .
$$

Recently, trace inequalities for the product of positive semidefinite matrices $A_{1}, A_{2}, \ldots, A_{m}$ in $M_{n}(\mathbb{C})$ were given in [6]:

$$
\operatorname{tr}\left|A_{1} A_{2} \cdots A_{m}\right| \leq \prod_{i=1}^{m}\left(\operatorname{tr} A_{i}^{p_{i}}\right)^{1 / p_{i}}
$$

and

$$
\operatorname{tr}\left|A_{1} A_{2} \cdots A_{m}\right| \leq \operatorname{tr}\left(\sum_{i=1}^{m} \frac{1}{p_{i}} A_{i}^{p_{i}}\right),
$$

where $p_{1}, \ldots, p_{m}$ are positive semidefinite real numbers such that $1 / p_{1}+\cdots+$ $1 / p_{m}=1$. A special case of (1.3) which generalises (1.2) is: if $A$ and $B$ are positive semidefinite matrices in $M_{n}(\mathbb{C})$ and $p, q$ are positive real numbers such that $1 / p+1 / q=1$, then

$$
\operatorname{tr}(A B) \leq\left(\operatorname{tr} A^{p}\right)^{1 / p}\left(\operatorname{tr} B^{q}\right)^{1 / q} .
$$

The main purpose of this paper is to establish trace inequalities for matrices. In Section 2 we invoke the majorisation relations for singular values and Hölder's inequality for positive real numbers to get a general trace inequality which yields some earlier results. In Section 3 we give trace inequalities for sums and powers of matrices.

\section{Trace inequalities for products of matrices}

In this section, new forms of Hölder and Young trace inequalities for matrices that generalise (1.3), (1.4) and (1.5) are given. The following result will be helpful in refining earlier results.

Theorem 2.1. Let $A_{i} \in M_{n}(\mathbb{C})$ and let $p_{i}$ be a positive real number $(i=1,2, \ldots, m)$ such that $\sum_{i=1}^{m} 1 / p_{i}=1$. Then

$$
\operatorname{tr}\left|\prod_{i=1}^{m} A_{i}\right|^{r} \leq \prod_{i=1}^{m}\left(\operatorname{tr}\left|A_{i}\right|^{r p_{i}}\right)^{1 / p_{i}} \quad \text { for } r \geq 1 .
$$


Proof. It is well known that the trace of a matrix equals the sum of its eigenvalues, so

$$
\operatorname{tr}\left|\prod_{i=1}^{m} A_{i}\right|^{r}=\sum_{j=1}^{n} \lambda_{j}\left(\left|\prod_{i=1}^{m} A_{i}\right|^{r}\right)=\sum_{j=1}^{n} \lambda_{j}^{r}\left(\left|\prod_{i=1}^{m} A_{i}\right|\right) .
$$

Since the singular values for any matrix are the eigenvalues of its absolute value,

$$
\sum_{j=1}^{n} \lambda_{j}^{r}\left(\left|\prod_{i=1}^{m} A_{i}\right|\right)=\sum_{j=1}^{n} s_{j}^{r}\left(\prod_{i=1}^{m} A_{i}\right)
$$

Using the weak majorisation of the product of singular values (see, for example, $[7$, p. 247]),

$$
\sum_{j=1}^{n} s_{j}^{r}\left(\prod_{i=1}^{m} A_{i}\right) \leq \sum_{j=1}^{n}\left(\prod_{i=1}^{m} s_{j}^{r}\left(A_{i}\right)\right) .
$$

Now Hölder's inequality for positive real numbers implies that

$$
\begin{aligned}
\sum_{j=1}^{n}\left(\prod_{i=1}^{m} s_{j}^{r}\left(A_{i}\right)\right) & \leq \prod_{i=1}^{m}\left(\sum_{j=1}^{n} s_{j}^{r p_{i}}\left(A_{i}\right)\right)^{1 / p_{i}}=\prod_{i=1}^{m}\left(\sum_{j=1}^{n} \lambda_{j}^{r p_{i}}\left(\left|A_{i}\right|\right)\right)^{1 / p_{i}} \\
& =\prod_{i=1}^{m}\left(\sum_{j=1}^{n} \lambda_{j}\left(\left|A_{i}\right|^{r p_{i}}\right)\right)^{1 / p_{i}}=\prod_{i=1}^{m}\left(\operatorname{tr}\left|A_{i}\right|^{r p_{i}}\right)^{1 / p_{i}} .
\end{aligned}
$$

This completes the proof.

A special case of (2.1) is the following. If $A, B \in M_{n}(\mathbb{C})$ and $p, q$ are positive real numbers such that $1 / p+1 / q=1$, then

$$
\operatorname{tr}|A B|^{r} \leq\left(\operatorname{tr}|A|^{r p}\right)^{1 / p}\left(\operatorname{tr}|B|^{r q}\right)^{1 / q},
$$

which generalises (1.5).

Inequality (2.1) can be considered as a Hölder trace inequality, while the Yong trace inequality can be stated as follows.

Corollary 2.2. Let $A_{i} \in M_{n}(\mathbb{C})$ and let $p_{i}$ be a positive real number $(i=1,2, \ldots, m)$ such that $\sum_{i=1}^{m} 1 / p_{i}=1$. Then

$$
\operatorname{tr}\left|\prod_{i=1}^{m} A_{i}\right|^{r} \leq \operatorname{tr}\left(\sum_{i=1}^{m} \frac{\left|A_{i}\right|^{r p_{i}}}{p_{i}}\right) \quad \text { for } r \geq 1
$$

Proof. Using (2.1) and Young's inequality for the positive real numbers $\operatorname{tr}\left|A_{1}\right|^{r p_{1}}$, $\operatorname{tr}\left|A_{2}\right|^{r p_{2}}, \ldots, \operatorname{tr}\left|A_{m}\right|^{r p_{m}}$,

$$
\operatorname{tr}\left|\prod_{i=1}^{m} A_{i}\right|^{r} \leq \prod_{i=1}^{m}\left(\operatorname{tr}\left|A_{i}\right|^{r p_{i}}\right)^{1 / p_{i}} \leq \sum_{i=1}^{m} \frac{1}{p_{i}} \operatorname{tr}\left|A_{i}\right|^{r p_{i}}=\operatorname{tr}\left(\sum_{i=1}^{m} \frac{\left|A_{i}\right|^{r p_{i}}}{p_{i}}\right),
$$

as required. 
Corollary 2.3. Let $A_{i} \in M_{n}(\mathbb{C})$ be positive semidefinite and $\alpha_{i}$ be a positive real number $(i=1,2, \ldots, m)$ such that $\sum_{i=1}^{m} \alpha_{i}=1$. Then, for $r \geq 1$,

$$
\operatorname{tr}\left|\prod_{i=1}^{m} A_{i}^{\alpha_{i}}\right|^{r} \leq \prod_{i=1}^{m}\left(\operatorname{tr} A_{i}^{r}\right)^{\alpha_{i}}
$$

and

$$
\operatorname{tr}\left|\prod_{i=1}^{m} A_{i}^{\alpha_{i}}\right|^{r} \leq \sum_{i=1}^{m} \alpha_{i} \operatorname{tr} A_{i}^{r}
$$

Proof. Replacing $A_{i}$ by $A_{i}^{\alpha_{i}}$ in (2.1) and letting $\alpha_{i}=1 / p_{i}(i=1,2, \ldots, m)$, we obtain (2.3). Inequality (2.4) follows from (2.3) and Young's inequality.

For $r=1$ in (2.3),

$$
\operatorname{tr}\left|\prod_{i=1}^{m} A_{i}^{\alpha_{i}}\right| \leq \prod_{i=1}^{m}\left(\operatorname{tr} A_{i}\right)^{\alpha_{i}}
$$

which improves a result of Chen and Wong [3], namely

$$
\left|\operatorname{tr} \prod_{i=1}^{m} A_{i}^{\alpha_{i}}\right| \leq \prod_{i=1}^{m}\left(\operatorname{tr} A_{i}\right)^{\alpha_{i}}
$$

Remark 2.4. Yang [11] presents the following conjecture. Let $A_{i}$ be a positive definite operator $(1 \leq i \leq m)$ in $C_{1}$. Does the inequality

$$
\left|\operatorname{tr}\left(A_{1} A_{2} \cdots A_{m}\right)\right|^{m} \leq \prod_{i=1}^{m} \operatorname{tr} A_{i}^{m}
$$

hold? For matrices the answer is yes. Indeed, for $r=1$ in (2.1),

$$
\operatorname{tr}\left|\prod_{i=1}^{m} A_{i}\right| \leq \prod_{i=1}^{m}\left(\operatorname{tr}\left|A_{i}\right|^{p_{i}}\right)^{1 / p_{i}}
$$

Letting $p_{i}=m(i=1,2, \ldots, m)$,

$$
\operatorname{tr}\left|A_{1} A_{2} \cdots A_{m}\right| \leq\left(\prod_{i=1}^{m} \operatorname{tr} A_{i}^{m}\right)^{1 / m}
$$

But

$$
\left|\operatorname{tr}\left(A_{1} A_{2} \cdots A_{m}\right)\right| \leq \operatorname{tr}\left|A_{1} A_{2} \cdots A_{m}\right|
$$

so

$$
\left|\operatorname{tr}\left(A_{1} A_{2} \cdots A_{m}\right)\right|^{m} \leq\left(\operatorname{tr}\left|A_{1} A_{2} \cdots A_{m}\right|\right)^{m} \leq \prod_{i=1}^{m} \operatorname{tr} A_{i}^{m} .
$$

A special case of (2.2) is the following. For positive semidefinite matrices $A$ and $B$,

$$
\operatorname{tr}\left|A B^{*}\right|^{r} \leq \operatorname{tr}\left(\frac{|A|^{r p}}{p}+\frac{|B|^{r q}}{q}\right)
$$


for positive real numbers $r, p$ and $q$ such that $r \geq 1$ and $1 / p+1 / q=1$. The following theorem introduces an inequality related to (2.5), but the relation between these two forms will be discussed in the next section.

THeorem 2.5. Let $A, B \in M_{n}(\mathbb{C})$ and $r$, $p$ and $q$ be positive real numbers such that $r \geq 1$ and $1 / p+1 / q=1$. Then

$$
\operatorname{tr}\left|A B^{*}\right|^{r} \leq \operatorname{tr}\left(\frac{|A|^{p}}{p}+\frac{|B|^{q}}{q}\right)^{r}
$$

Proof. Since the trace of a matrix is equal to the sum of its eigenvalues,

$$
\operatorname{tr}\left|A B^{*}\right|^{r}=\sum_{j=1}^{n} \lambda_{j}\left(\left|A B^{*}\right|^{r}\right)=\sum_{j=1}^{n} \lambda_{j}^{r}\left(\left|A B^{*}\right|\right)=\sum_{j=1}^{n} s_{j}^{r}\left(A B^{*}\right) .
$$

By using the matrix version of Young's inequality (see, for example, [14]), we have, for $j=1,2, \ldots, n$,

$$
s_{j}\left(A B^{*}\right) \leq s_{j}\left(\frac{|A|^{p}}{p}+\frac{|B|^{q}}{q}\right) \text {. }
$$

Hence

$$
\begin{aligned}
\operatorname{tr}\left|A B^{*}\right|^{r} & \leq \sum_{j=1}^{n} s_{j}^{r}\left(\frac{|A|^{p}}{p}+\frac{|B|^{q}}{q}\right)=\sum_{j=1}^{n} s_{j}\left(\left(\frac{|A|^{p}}{p}+\frac{|B|^{q}}{q}\right)^{r}\right) \\
& =\sum_{j=1}^{n} \lambda_{j}\left(\left(\frac{|A|^{p}}{p}+\frac{|B|^{q}}{q}\right)^{r}\right)=\operatorname{tr}\left(\frac{|A|^{p}}{p}+\frac{|B|^{q}}{q}\right)^{r} .
\end{aligned}
$$

This completes the proof.

For $p=q=2$ and $r=1$ in (2.6), we have the arithmetic-geometric trace inequality

$$
\operatorname{tr}\left|A B^{*}\right| \leq \frac{1}{2} \operatorname{tr}\left(A^{*} A+B^{*} B\right) .
$$

The following theorem depends on Hölder's inequality and singular value majorisation to get a trace inequality for a sum of matrices.

Theorem 2.6. Let $A_{i}, B_{i} \in M_{n}(\mathbb{C})(i=1,2, \ldots, m)$ be positive semidefinite matrices and $p, q$ be positive real numbers such that $1 / p+1 / q=1$. Then

$$
\operatorname{tr}\left(\sum_{i=1}^{m} A_{i} B_{i}\right) \leq\left(\operatorname{tr}\left(\sum_{i=1}^{m} A_{i}^{p}\right)\right)^{1 / p}\left(\operatorname{tr}\left(\sum_{i=1}^{m} B_{i}^{q}\right)\right)^{1 / q} .
$$

In particular,

$$
\left(\operatorname{tr}\left(\sum_{i=1}^{m} A_{i} B_{i}\right)\right)^{2} \leq\left(\operatorname{tr}\left(\sum_{i=1}^{m} A_{i}^{2}\right)\right)\left(\operatorname{tr}\left(\sum_{i=1}^{m} B_{i}^{2}\right)\right)
$$


Proof. Since the trace functional is linear,

$$
\operatorname{tr}\left(\sum_{i=1}^{m} A_{i} B_{i}\right)=\sum_{i=1}^{m} \operatorname{tr}\left(A_{i} B_{i}\right)=\sum_{i=1}^{m} \sum_{j=1}^{n} \lambda_{j}\left(A_{i} B_{i}\right)=\sum_{i=1}^{m}\left(\sum_{j=1}^{n}\left|\lambda_{j}\left(A_{i} B_{i}\right)\right|\right) .
$$

The last equality follows from the fact that the eigenvalues of the product of positive semidefinite matrices are positive. Since the modulus of the eigenvalues of any matrix are weak majorised by its singular values,

$$
\begin{aligned}
\sum_{i=1}^{m}\left(\sum_{j=1}^{n}\left|\lambda_{j}\left(A_{i} B_{i}\right)\right|\right) & \leq \sum_{i=1}^{m}\left(\sum_{j=1}^{n} s_{j}\left(A_{i} B_{i}\right)\right)=\sum_{j=1}^{n}\left(\sum_{i=1}^{m} s_{j}\left(A_{i} B_{i}\right)\right) \\
& \leq \sum_{j=1}^{n}\left(\sum_{i=1}^{m} s_{j}\left(A_{i}\right) s_{j}\left(B_{i}\right)\right)=\sum_{i=1}^{m}\left(\sum_{j=1}^{n} s_{j}\left(A_{i}\right) s_{j}\left(B_{i}\right)\right) .
\end{aligned}
$$

Using Hölder's inequality for the nonnegative real numbers $s_{1}\left(A_{i}\right), s_{2}\left(A_{i}\right), \ldots, s_{n}\left(A_{i}\right)$ and $s_{1}\left(B_{i}\right), s_{2}\left(B_{i}\right), \ldots, s_{n}\left(B_{i}\right)$,

$$
\begin{aligned}
\sum_{j=1}^{n} s_{j}\left(A_{i}\right) s_{j}\left(B_{i}\right) & \leq\left(\sum_{j=1}^{n} s_{j}^{p}\left(A_{i}\right)\right)^{1 / p}\left(\sum_{j=1}^{n} s_{j}^{q}\left(B_{i}\right)\right)^{1 / q} \\
& =\left(\sum_{j=1}^{n} s_{j}\left(A_{i}^{p}\right)\right)^{1 / p}\left(\sum_{j=1}^{n} s_{j}\left(B_{i}^{q}\right)\right)^{1 / q} \\
& =\left(\operatorname{tr}\left(A_{i}^{p}\right)\right)^{1 / p}\left(\operatorname{tr}\left(B_{i}^{q}\right)\right)^{1 / q}
\end{aligned}
$$

Hence,

$$
\operatorname{tr}\left(\sum_{i=1}^{m} A_{i} B_{i}\right) \leq \sum_{i=1}^{m}\left(\operatorname{tr}\left(A_{i}^{p}\right)\right)^{1 / p}\left(\operatorname{tr}\left(B_{i}^{q}\right)\right)^{1 / q} .
$$

Again using Hölder's inequality for real numbers,

$$
\operatorname{tr}\left(\sum_{i=1}^{m} A_{i} B_{i}\right) \leq\left(\sum_{i=1}^{m} \operatorname{tr}\left(A_{i}^{p}\right)\right)^{1 / p}\left(\sum_{i=1}^{m} \operatorname{tr}\left(B_{i}^{q}\right)\right)^{1 / q},
$$

and the proof is complete.

Using Young's inequality and (2.5), we have the following corollary.

Corollary 2.7. Let $A_{i}, B_{i} \in M_{n}(\mathbb{C})(i=1,2, \ldots, m)$ be positive semidefinite matrices and $p, q$ be positive real numbers such that $1 / p+1 / q=1$. Then

$$
\operatorname{tr}\left(\sum_{i=1}^{m} A_{i} B_{i}\right) \leq \operatorname{tr}\left(\frac{1}{p} \sum_{i=1}^{m} A_{i}^{p}+\frac{1}{q} \sum_{i=1}^{m} B_{i}^{q}\right) .
$$

We end this section with the following theorem.

Theorem 2.8. Let $A_{i}, B_{i} \in M_{n}(\mathbb{C})(i=1,2, \ldots, m)$ be positive semidefinite matrices and $p, q$ be positive real numbers such that $1 / p+1 / q=1$. Then, for any natural 
number $k$,

$$
\operatorname{tr}\left(\sum_{i=1}^{m}\left(A_{i} B_{i}\right)^{k}\right) \leq\left(\sum_{i=1}^{m}\left\|A_{i}\right\|^{p k}\right)^{1 / p}\left(\sum_{i=1}^{m}\left(\operatorname{tr} B_{i}^{k}\right)^{q}\right)^{1 / q}
$$

and

$$
\operatorname{tr}\left(\sum_{i=1}^{m}\left(A_{i} B_{i}\right)^{k}\right) \leq\left(\sum_{i=1}^{m}\left\|B_{i}\right\|^{p k}\right)^{1 / p}\left(\sum_{i=1}^{m}\left(\operatorname{tr} A_{i}^{k}\right)^{q}\right)^{1 / q} .
$$

In particular, if $A$ and $B$ are positive semidefinite matrices then

$$
\operatorname{tr}(A B)^{k} \leq \min \left\{\|A\|^{k} \operatorname{tr} B^{k},\|B\|^{k} \operatorname{tr} A^{k}\right\} .
$$

Proof. Using the linearity of the trace,

$$
\operatorname{tr}\left(\sum_{i=1}^{m}\left(A_{i} B_{i}\right)^{k}\right)=\sum_{i=1}^{m} \operatorname{tr}\left(A_{i} B_{i}\right)^{k}=\sum_{i=1}^{m} \sum_{j=1}^{n} \lambda_{j}\left(\left(A_{i} B_{i}\right)^{k}\right)=\sum_{i=1}^{m} \sum_{j=1}^{n} \lambda_{j}^{k}\left(A_{i} B_{i}\right) .
$$

But the eigenvalues of $A_{i} B_{i}$ are positive $(i=1,2, \ldots, m)$, so

$$
\sum_{i=1}^{m} \sum_{j=1}^{n} \lambda_{j}^{k}\left(A_{i} B_{i}\right)=\sum_{i=1}^{m} \sum_{j=1}^{n}\left|\lambda_{j}^{k}\left(A_{i} B_{i}\right)\right| \leq \sum_{i=1}^{m} \sum_{j=1}^{n} s_{j}^{k}\left(A_{i} B_{i}\right) .
$$

Since

$$
s_{j}\left(A_{i} B_{i}\right) \leq\left\|A_{i}\right\| s_{j}\left(B_{i}\right)
$$

for $j=1,2, \ldots, n$,

$$
\sum_{i=1}^{m} \sum_{j=1}^{n} \lambda_{j}^{k}\left(A_{i} B_{i}\right) \leq \sum_{i=1}^{m} \sum_{j=1}^{n}\left\|A_{i}\right\|^{k} s_{j}^{k}\left(B_{i}\right)=\sum_{i=1}^{m}\left(\left\|A_{i}\right\|^{k} \sum_{j=1}^{n} s_{j}^{k}\left(B_{i}\right)\right) .
$$

Now,

$$
\sum_{j=1}^{n} s_{j}^{k}\left(B_{i}\right)=\sum_{j=1}^{n} \lambda_{j}^{k}\left(B_{i}\right)=\sum_{j=1}^{n} \lambda_{j}\left(B_{i}^{k}\right)=\operatorname{tr} B_{i}^{k} .
$$

Using Hölder's inequality for positive real numbers,

$$
\begin{aligned}
\sum_{i=1}^{m} \sum_{j=1}^{n} \lambda_{j}^{k}\left(A_{i} B_{i}\right) & \leq \sum_{i=1}^{m}\left(\left\|A_{i}\right\|^{k}\right)\left(\operatorname{tr} B_{i}^{k}\right) \\
& \leq\left(\sum_{i=1}^{m}\left\|A_{i}\right\|^{p k}\right)^{1 / p}\left(\sum_{i=1}^{m}\left(\operatorname{tr} B_{i}^{k}\right)^{q}\right)^{1 / q} .
\end{aligned}
$$

Now, (2.7) follows from (2.10) and (2.12). If, instead of (2.11), we use the inequality

$$
s_{j}\left(A_{i} B_{i}\right) \leq\left\|B_{i}\right\| s_{j}\left(A_{i}\right),
$$

we obtain (2.8).

Inequality (2.9) improves the famous trace inequality (1.1). For further discussion of the submultiplicativity of the trace functional see $[4,12,13]$. 


\section{Trace inequalities for the sum of matrices}

This section introduces an important trace inequality that helps us to compare inequalities (2.5) and (2.6). Related inequalities are also presented.

Theorem 3.1. Let $A_{i} \in M_{n}(\mathbb{C})(i=1,2, \ldots, m)$ and $r \geq 1$. Then

$$
\operatorname{tr}\left|\sum_{i=1}^{m} A_{i}\right|^{r} \leq m^{r-1} \operatorname{tr}\left(\sum_{i=1}^{m}\left|A_{i}\right|^{r}\right) \text {. }
$$

In particular, for $A, B \in M_{n}(\mathbb{C})$,

$$
\operatorname{tr}|A+B| \leq \operatorname{tr}(|A|+|B|) .
$$

Proof. Using the fact that the trace of a matrix is equal to the sum of the eigenvalues,

$$
\operatorname{tr}\left|\sum_{i=1}^{m} A_{i}\right|^{r}=\sum_{j=1}^{n} \lambda_{j}\left(\left|\sum_{i=1}^{m} A_{i}\right|^{r}\right)=\sum_{j=1}^{n} \lambda_{j}^{r}\left(\left|\sum_{i=1}^{m} A_{i}\right|\right)=\sum_{j=1}^{n} s_{j}^{r}\left(\sum_{i=1}^{m} A_{i}\right) .
$$

By the Fan singular value majorisation theorem [7, p. 243],

$$
\sum_{j=1}^{k} s_{j}\left(A_{1}+A_{2}+\cdots+A_{m}\right) \leq \sum_{j=1}^{k}\left(s_{j}\left(A_{1}\right)+s_{j}\left(A_{2}\right)+\cdots+s_{j}\left(A_{m}\right)\right)
$$

for $k=1,2, \ldots, n$. Hence, for any increasing function $f$ on $[0, \infty)$,

$$
\sum_{j=1}^{n} f\left(s_{j}\left(A_{1}+A_{2}+\cdots+A_{m}\right)\right) \leq \sum_{j=1}^{n} f\left(s_{j}\left(A_{1}\right)+s_{j}\left(A_{2}\right)+\cdots+s_{j}\left(A_{m}\right)\right) .
$$

In particular,

$$
\begin{aligned}
\sum_{j=1}^{n} s_{j}^{r}\left(A_{1}+A_{2}+\cdots+A_{m}\right) & \leq \sum_{j=1}^{n}\left(s_{j}\left(A_{1}\right)+s_{j}\left(A_{2}\right)+\cdots+s_{j}\left(A_{m}\right)\right)^{r} \\
& \leq m^{r-1} \sum_{j=1}^{n}\left(s_{j}^{r}\left(A_{1}\right)+s_{j}^{r}\left(A_{2}\right)+\cdots+s_{j}^{r}\left(A_{m}\right)\right) \\
& =m^{r-1} \sum_{j=1}^{n}\left(\lambda_{j}^{r}\left(\left|A_{1}\right|\right)+\lambda_{j}^{r}\left(\left|A_{2}\right|\right)+\cdots+\lambda_{j}^{r}\left(\left|A_{m}\right|\right)\right) \\
& =m^{r-1} \sum_{j=1}^{n}\left(\lambda_{j}\left(\left|A_{1}\right|^{r}\right)+\lambda_{j}\left(\left|A_{2}\right|^{r}\right)+\cdots+\lambda_{j}\left(\left|A_{m}\right|^{r}\right)\right) \\
& =m^{r-1} \operatorname{tr}\left(\left|A_{1}\right|^{r}+\left|A_{2}\right|^{r}+\cdots+\left|A_{m}\right|^{r}\right) .
\end{aligned}
$$

This completes the proof. 
In view of (3.2), it is known that the inequality

$$
\||A+B||| \leq|||A|+|B|||
$$

does not hold in general for arbitrary matrices $A$ and $B$ in $M_{n}(\mathbb{C})$ (see, for example, [2, p. 27]). But (3.3) holds for normal matrices $A$ and $B$ in $M_{n}(\mathbb{C})$. It should be mentioned here that a generalisation of (3.3) for normal matrices was given in [9].

Corollary 3.2. Let $A, B \in M_{n}(\mathbb{C})$ and $r \geq 1$. Then

$$
\operatorname{tr}\left(|A|^{r}+|B|^{r}\right) \leq \operatorname{tr}\left(|A+B|^{r}+|A-B|^{r}\right) .
$$

Proof. Let $A_{1}=A, A_{2}=B$ and $m=2$ in (3.1). Then

$$
\operatorname{tr}|A+B|^{r} \leq 2^{r-1} \operatorname{tr}\left(|A|^{r}+|B|^{r}\right) .
$$

Replacing $A$ by $A+B$ and $B$ by $A-B$,

$$
\operatorname{tr}|A|^{r} \leq \frac{1}{2} \operatorname{tr}\left(|A+B|^{r}+|A-B|^{r}\right) .
$$

Again, replacing $A$ by $A+B$ and $B$ by $B-A$,

$$
\operatorname{tr}|B|^{r} \leq \frac{1}{2} \operatorname{tr}\left(|A+B|^{r}+|A-B|^{r}\right) .
$$

Adding (3.4) and (3.5), we get the result.

We end this paper with the following remark.

REMARK 3.3. Using (3.1) we can compare the two inequalities (2.5) and (2.6). For the case $p=q=2$,

$$
\operatorname{tr}\left(\frac{|A|^{2}+|B|^{2}}{2}\right)^{r} \leq 2^{r-1} \operatorname{tr}\left(\left(\frac{|A|^{2}}{2}\right)^{r}+\left(\frac{|B|^{2}}{2}\right)^{r}\right)=\operatorname{tr}\left(\frac{|A|^{2 r}}{2}+\frac{|B|^{2 r}}{2}\right),
$$

so, in this case, (2.6) is better than (2.5). It should be mentioned here that related inequalities for unitarily invariant norms were presented in [8].

\section{Acknowledgements}

This work was done while the first author was at Qassim University, Saudi Arabia on sabbatical leave from Al-Balqa' Applied University, Jordan. The author thanks both universities for their support.

\section{References}

[1] T. Ando, 'Matrix Young inequalities', Oper. Theory Adv. Appl. 75 (1995), 33-38.

[2] R. Bhatia, Positive Definite Matrices (Princeton University Press, Princeton, NJ, 2007).

[3] L. Chen and C. Wong, 'Inequalities for singular values and traces', Linear Algebra Appl. 171 (1992), 109-120. 
[4] I. D. Coope, 'On matrix trace inequalities and related topics for products of Hermitian matrices', J. Math. Anal. Appl. 188 (1994), 999-1001.

[5] J. Fujii, 'A trace inequality arising from quantum information theory', Linear Algebra Appl. 400 (2005), 141-146.

[6] S. Manjegani, 'Hölder and Young inequalities for the trace of operators', Positivity 11 (2007), 239-250.

[7] A. W. Marshall and I. Olkin, Inequalities: Theory of Majorization and its Applications (Academic Press, San Diego, CA, 1979).

[8] K. Shebrawi and H. Albadawi, 'Operator norm inequalities of Minkowski type', J. Inequal. Pure Appl. Math. 9(1) (2008), 1-10, article 26.

[9] K. Shebrawi and H. Albadawi, 'Numerical radius and operator norm inequalities', J. Inequal. Appl. 2009 article 492154.

[10] B. Simon, Trace Ideals and Their Applications (Cambridge University Press, Cambridge, 1979).

[11] X. Yang, 'Some trace inequalities for operators', J. Aust. Math. Soc. A 58 (1995), 281-286.

[12] X. Yang, 'A matrix trace inequality', J. Math. Anal. Appl. 250 (2000), 372-374.

[13] X. M. Yang, X. Q. Yang and K. L. Teo, 'A matrix trace inequality', J. Math. Anal. Appl. 263 (2001), 327-331.

[14] X. Zhan, Matrix Inequalities (Springer, Berlin, 2002).

KHALID SHEBRAWI, Department of Mathematics, Faculty of Science, Al-Balqa' Applied University, Salt, Jordan

and

Department of Mathematics, Faculty of Science, Qassim University,

Qassim, Saudi Arabia

e-mail: shebrawi@gmail.com

HUSSIEN ALBADAWI, Preparatory Year Deanship, King Faisal University, Ahsaa, Saudi Arabia

e-mail: halbadawi@kfu.edu.sa 